\title{
EXPONENTIAL ASYMPTOTICS FOR AN EIGENVALUE OF A PROBLEM INVOLVING PARABOLIC CYLINDER FUNCTIONS.
}

\author{
NEAL BRAZEL, FIONA LAWLESS, AND ALASTAIR WOOD
}

(Communicated by Kenneth R. Meyer)

\begin{abstract}
We obtain the leading asymptotic behaviour as $\varepsilon \rightarrow 0+$ of the exponentially small imaginary part of the "eigenvalue" of the perturbed nonself-adjoint problem comprising $y^{\prime \prime}(x)+\left(\lambda+\varepsilon x^{2}\right) y(x)=0$ with a linear homogeneous boundary condition at $x=0$ and an "outgoing wave" condition as $x \rightarrow+\infty$. The problem is a generalization of a model equation for optical tunnelling considered by Paris and Wood [10]. We show that this "eigenvalue" corresponds to a pole in the Titchmarsh-Weyl function $m(\lambda)$ for the corresponding formally self-adjoint problem with $L^{2}(0, \infty)$ boundary condition.
\end{abstract}

\section{INTRODUCTION}

Let $\varepsilon \geq 0$ and $h$ by a positive constant. We consider the problem of finding the imaginary part of the eigenvalue $\lambda$ of the differential equation

$$
y^{\prime \prime}(x)+\left(\lambda+\varepsilon x^{2}\right) y(x)=0 \quad \text { on }(0, \infty)
$$

with

$$
y^{\prime}(0)+h y(0)=0,
$$

together with the condition that $y(x)$ has a controlling behaviour $\exp (\operatorname{ip}(x))$ as $x \rightarrow+\infty$ for some positive real-valued function $p(x)$. There is a unique function $p(x)$ with this property. We determine it by the Liouville-Green approximation. This seeks the controlling behaviour of the solution as $x \rightarrow+\infty$ via the substitution $y(x)=e^{\mathrm{ip}(x)}$, which yields the result $p(x)=\frac{1}{2} \varepsilon^{1 / 2} x^{2}$ for this problem. We may write this boundary condition in a Wronskian format more familiar in spectral theory as

$$
\lim _{X \rightarrow \infty} W\left[y(X), e^{\frac{i}{2} \varepsilon^{1 / 2} X^{2}}\right]=0 .
$$

It is easy to show that the unperturbed eigenvalue is at $\lambda=-h^{2}$ and that the perturbed eigenvalue problem is not self-adjoint because of the form of the

Received by the editors October 7, 1990.

1980 Mathematics Subject Classification (1985 Revision). Primary 34B20, 34B25, 34E20; Secondary 33A15, 33A70.

Key words and phrases. Exponential asymptotics, singular perturbation, spectral theory, Titchmarsh-Weyl coefficient, parabolic cylinder functions.

The second author is supported by an Eolas Applied Research and Development Scholarship. 
boundary condition at infinity; the proof follows the same lines as $\S 3$ of [10]. The simpler equation with potential $\varepsilon x$ instead of $\varepsilon x^{2}$ was studied by Paris and Wood [10] as a model equation for optical tunnelling arising from work by Kath and Kriegsmann [3]. In that application, the imaginary part of the perturbed eigenvalue represents the rate at which energy leaks out of the core of bent fibre optics waveguides; for details see Kath and Kriegsmann [3]. The boundary condition at infinity for (1.1) arises from the outgoing wave condition. Certain technical difficulties with Stokes' discontinuities (see Berry [2] and Olver [8]) were encountered in [10] where the imaginary part of the eigenvalue was found to be

$$
\operatorname{Im} \lambda \sim \frac{-2 h^{2}}{e} \exp \left[\frac{-4 h^{3}}{3 \varepsilon}\right] \quad \text { as } \varepsilon \rightarrow 0+,
$$

compared to the eigenvalue itself

$$
\lambda \sim-h^{2}-\frac{\varepsilon}{2 h}-\frac{\varepsilon^{2}}{8 h^{4}}+O\left(\varepsilon^{3}\right) \quad \text { as } \varepsilon \rightarrow 0+.
$$

Thus we are in an area of exponential asymptotics described by Meyer [6].

The physical problem treated in [3] and [10] essentially is one of radiation damping. This is a difficult area of transcendental asymptotics that has yet to be given a satisfactory general treatment, even for ordinary differential equations. For a different analysis of another ordinary differential equation arising in the theory of surface waves trapped by round islands of small seabed slope, see the paper by Lozano and Meyer [5]. In their case, the equation is too complicated to treat using special functions, and they become involved with the details of connecting WKB solutions across transition points. Our result relies on finding an exact solution of (1.1) in terms of functions whose exponential asymptotics may be constructed from known results.

We shall prove in this paper that similar exponentially small phenomena occur for the equation (1.1) and that

$$
\operatorname{Im} \lambda \sim-2 h^{2} \exp \left[\frac{-h^{2} \pi}{2 \sqrt{\varepsilon}}\right] \quad \text { as } \varepsilon \rightarrow 0+.
$$

This result will be established by two different methods. For both, a knowledge of the asymptotics of the parabolic cylinder function will be required. These results are assembled in $\S 2$, where we also establish exponentially improved asymptotics of a quotient of gamma functions.

The direct method in $\S 3$ clearly follows the development in [10]. Knowledge of the asymptotics of the parabolic cylinder functions enables us to construct a linear combination of such solutions that satisfies the outgoing wave condition at infinity. Insertion of this combination in the boundary condition at the origin, if manipulated with due respect for the exponentially small terms, yields the expression (1.3).

The complex number $\lambda$ obtained by the direct method is an eigenvalue in the sense that the differential equation (1.1) possesses a nontrivial solution satisfying both boundary conditions when this value of $\lambda$ is taken. It has no direct interpretation in operator theory. To provide a more abstract setting for this result, we must go to quantum mechanics and the theory of resonances or "pseudo-eigenvalues" as found in the opening chapter of Reed and Simon [11]. 
These resonances correspond to poles of the Titchmarsh-Weyl $m(\lambda)$ function situated just below the negative real axis; for a classical treatment see Titchmarsh [13, Chapter XX], although the word "resonance" is not mentioned there. It is shown in a separate paper by the second and third authors [4] that, for a more general class of potentials including (1.1), the complex eigenvalues of the non-self-adjoint problem here correspond to the resonance poles of the self-adjoint problem where the outgoing wave condition is replaced by an $L^{2}$ boundary condition at $+\infty$. Accordingly, in $\S 4$, we construct the $m(\lambda)$ function using results in [12, Chapter IV] and determine its pole approximated by (1.3).

\section{SOME ASYMPTOTIC PREREQUISITIES}

In this section we construct a suitable fundamental set of solutions for (1.1) in terms of Weber parabolic cylinder functions and give their asymptotic expansions. We also obtain exponentially improved asymptotics for a quotient of gamma functions that will be required in $\S 3$. If in (1.1) we make the transformations $z=e^{\frac{i \pi}{4}} 2^{\frac{1}{2}} \varepsilon^{\frac{1}{4}} x$ and $a=\frac{1}{2} \varepsilon^{-1 / 2} i \lambda$, we obtain the equation

$$
\frac{d^{2} y}{d x^{2}}=\left(a+\frac{1}{4} z^{2}\right) y
$$

with boundary condition

$$
e^{\frac{i \pi}{4}} 2^{\frac{1}{2}} \varepsilon^{\frac{1}{4}} \frac{d y(0)}{d x}+h y(0)=0
$$

and the same "outgoing wave" condition at infinity (but note that $z \rightarrow \infty e^{i \pi / 4}$ as $x \rightarrow+\infty)$. It will be convenient to work with both the solution sets $U(a, z), V(a, z)$, and Whittaker's parabolic cylinder functions $D_{-a-1 / 2}(z)$, $D_{a-1 / 2}(-i z)$ (see Abramowitz and Stegun [1, p. 687]) that are related by the formula

$$
U(a, z)=D_{-a-1 / 2}(z) .
$$

$U(a, z)$ is Weber's parabolic cylinder function defined as the solution of $(2.1)$ with leading behaviour

$$
U(a, z) \sim z^{-a-\frac{1}{2}} e^{-z^{2} / 4}
$$

as $z \rightarrow \infty$ in the sector containing the positive real axis. $V(a, z)$ is then defined by

$$
V(a, z)=\frac{1}{\pi} \Gamma\left(\frac{1}{2}+a\right)\{(\sin \pi a) U(a, z)+U(a,-z)\} .
$$

For fixed $a$ and large $|z|$, we see from [8] that

$$
U(a, z) \sim z^{-a-\frac{1}{2}} e^{-\frac{z^{2}}{4}}\left[1+O\left(|z|^{-2}\right)\right], \quad|\arg z|<3 \pi / 4,
$$

while from the connection formulae between $U(a, \pm z)$ and $U(-a, \pm i z)$ (see [1]) it can easily be seen that

$$
\begin{aligned}
& U(a,-z) \sim e^{i \pi\left(a+\frac{1}{2}\right)} z^{-a-\frac{1}{2}} e^{\frac{-z^{2}}{4}}\left[1+O\left(|z|^{-2}\right)\right] \\
& \quad+\frac{\sqrt{2 \pi}}{\Gamma\left(\frac{1}{2}+a\right)} z^{a-\frac{1}{2}} e^{\frac{z^{2}}{4}}\left[1+O\left(|z|^{-2}\right)\right], \quad 0<\arg z<\frac{\pi}{2} .
\end{aligned}
$$


These results enable us to construct the following "outgoing wave" solution from the fundamental solutions $U(a, z)$ and $V(a, z)$,

$$
y(z)=\frac{1}{\pi} \Gamma\left(\frac{1}{2}+a\right)\left[U(a,-z)-i e^{i \pi a} U(a, z)\right] .
$$

Although this solution ensures that the leading terms associated with $e^{-z^{2} / 4}$ cancel and that we have an "outgoing wave" solution to leading behaviour, it is not obvious what happened to lower terms in $e^{-z^{2} / 4}$. This can be resolved by means of the connection formula

$$
U(a,-z)=\frac{\sqrt{2 \pi}}{\Gamma\left(\frac{1}{2}+a\right)} e^{i \pi\left(\frac{a}{2}-\frac{1}{4}\right)} U(-a,-i z)-e^{2 i \pi\left(\frac{a}{2}-\frac{1}{4}\right)} U(a, z)
$$

which reduces $(2.8)$ to

$$
y(z)=\left(\frac{2}{\pi}\right)^{1 / 2} e^{\frac{1}{2} i \pi\left(a-\frac{1}{2}\right)} U(-a,-i z),
$$

where the asymptotic expansion $U(-a,-i z)$ contains terms involving only $e^{z^{2} / 4}$ as $z \rightarrow \infty$ in $0<\arg z<\pi / 2$.

We observe that the equation with potential $\varepsilon x^{2}$ differs from that with potential $\varepsilon x$ considered in [10] in that it is not necessary to use the results of Berry [2] and Olver [9] in the neighbourhood of Stokes lines.

The second objective in this section is to derive exponentially improved asymptotic expansions for a quotient of gamma functions as $z \rightarrow \infty$ close to the negative imaginary axis. The result (5.02) of Olver [7] contains only algebraic terms and is insufficiently precise for our purposes in $\S \S 3,4$. We shall derive the result for this required special case $\Gamma\left(\frac{1}{4}+z\right) / \Gamma\left(\frac{3}{4}+z\right)$, although the method holds for $\Gamma(\alpha+z) / \Gamma(\beta+z)$ for any $\alpha, \beta \in \mathscr{C}$ such that $\alpha+\beta=1$. By the reflection formula, we may rearrange the quotient as

$$
\frac{\Gamma\left(\frac{1}{4}+z\right)}{\Gamma\left(\frac{3}{4}+z\right)}=\frac{1}{\pi} \Gamma\left(\frac{1}{4}+z\right) \Gamma\left(\frac{1}{4}-z\right) \sin \pi\left(\frac{1}{4}-z\right) .
$$

Writing $z=i y, y \in \mathscr{R}$ and using the result $\Gamma(\bar{z})=\overline{\Gamma(z)}$,

$$
\begin{aligned}
\frac{\Gamma\left(\frac{1}{4}+z\right)}{\Gamma\left(\frac{3}{4}+z\right)} & =\frac{1}{\pi}\left|\Gamma\left(\frac{1}{4}+i y\right)\right|^{2} \sin \pi\left(\frac{1}{4}-i y\right) \\
& \sim-i|y|^{-1 / 2} e^{-\pi|y|}\left\{e^{i \pi(1 / 4-i y)}-e^{-i \pi(1 / 4-i y)}\right\} \quad \text { as }|y| \rightarrow \infty
\end{aligned}
$$

on employing Stirling's approximation. For $y=|y|(z=i|y|)$ we find that

$$
\frac{\Gamma\left(\frac{1}{4}+z\right)}{\Gamma\left(\frac{3}{4}+z\right)} z^{-1 / 2}\left(1+i e^{2 i \pi z}\right)
$$

while for $y=-|y|(z=-i|y|)$,

$$
\frac{\Gamma\left(\frac{1}{4}+z\right)}{\Gamma\left(\frac{3}{4}+z\right)} \sim z^{-1 / 2}\left(1-i e^{-2 i \pi z}\right)
$$

Higher order real and imaginary terms could be obtained by using the full series expansion in Stirling's formula. 
This result is also valid in a small sector to the right of the imaginary axis. To see this, let $z=i y+\delta$, where $\delta=O\left(e^{-2 \pi|y|}\right)$. Using Taylor's expansion about $\delta=0$, we have

$$
\frac{\Gamma\left(\frac{1}{4}+(z+\delta)\right)}{\Gamma\left(\frac{3}{4}+(z+\delta)\right)}=\frac{\Gamma\left(\frac{1}{4}+z\right)}{\Gamma\left(\frac{3}{4}+z\right)}\left[1+\delta\left\{\psi\left(\frac{1}{4}+z\right)-\psi\left(\frac{3}{4}+z\right)\right\}+O\left(\delta^{2}\right)\right]
$$

where, as usual, $\psi$ denotes the logarithmic derivative of $\Gamma$. By means of the expansion [1, p. 259],

$$
\psi(z) \sim \log z-1 / 2 z-\ldots, \quad \text { as } z \rightarrow \infty \text { in }|\arg z|<\pi,
$$

(2.12) may be reduced to

$$
\frac{\Gamma\left(\frac{1}{4}+(z+\delta)\right)}{\Gamma\left(\frac{3}{4}+(z+\delta)\right)}=\frac{\Gamma\left(\frac{1}{4}+z\right)}{\Gamma\left(\frac{3}{4}+z\right)}\left[1+\frac{i \delta}{2 y}+O\left(\delta^{2}\right)\right] .
$$

But $i \delta / 2 y$ is $O\left(e^{-2 \pi|y|} /|y|\right)$, which may be neglected in (2.11). Thus (2.11) remains valid when $z$ is replaced by $z+\delta$ for sufficiently small $\delta$. This will be adequate for our purposes in later sections. The question of exponential improvement of Olver's result (5.02) for general $z$ remains open and will be addressed elsewhere.

\section{THE DIRECT METHOD FOR THE NON-SELF-ADJOINT PROBLEM}

Now we substitute the outgoing wave solution (2.8) of the transformed differential equation (2.1) into the transformed boundary condition at the origin (2.2) to obtain

$$
\left.e^{\frac{i \pi}{4}} 2^{\frac{1}{2}} \varepsilon^{\frac{1}{4}}\left\{-U^{\prime}(a, 0)-i e^{i a \pi} U^{\prime}(a, 0)\right)\right\}+h\left\{U(a, 0)-i e^{i a \pi} U(a, 0)\right\}=0,
$$

which we rearrange as

$$
\frac{U(a, 0)}{U^{\prime}(a, 0)}=\frac{e^{\frac{i \pi}{4}} 2^{\frac{1}{2}} \varepsilon^{\frac{1}{4}}\left(1+i e^{i a \pi}\right)}{h\left(1-i e^{i a \pi}\right)}
$$

which becomes

$$
\frac{\Gamma\left(\frac{1}{4}+\frac{a}{2}\right)}{\Gamma\left(\frac{3}{4}+\frac{a}{2}\right)}=-\frac{2 e^{i \frac{i \pi}{4}} \varepsilon^{\frac{1}{4}}\left(1+i e^{i a \pi}\right)}{h\left(1-i e^{i a \pi}\right)}
$$

on inserting the values of the parabolic cylinder function and its derivatives at the origin in (19.3.5) of [1]. Recall from (2.1) that $a=\frac{1}{2} \varepsilon^{-1 / 2} i \lambda$. It can be shown by a process of regular perturbation, similarly to (1.2), that

$$
\lambda=-h^{2}-\frac{\varepsilon}{2 h^{2}}-\frac{7 \varepsilon^{2}}{8 h^{6}}-\frac{121 \varepsilon^{3}}{16 h^{10}}+O\left(\varepsilon^{4}\right)
$$

and that the coefficient of every power $\varepsilon^{n}, n \in \mathscr{N}$ is real.

We conclude that $\operatorname{Im} \lambda<<\varepsilon^{n}$ for all $n \in \mathscr{N}$, and hence $\operatorname{Im} \lambda$ must be transcendentally small as $\varepsilon \rightarrow 0+$. It follows that $a \rightarrow-i \infty$ as $\varepsilon \rightarrow 0+$, in such a way as to be able to apply the exponentially improved approximation (2.11) to the left-hand side of (3.3). We find that

$$
\frac{\Gamma\left(\frac{1}{4}+\frac{a}{2}\right)}{\Gamma\left(\frac{3}{4}+\frac{a}{2}\right)} \sim\left(\frac{a}{2}\right)^{-1 / 2}\left(1-i e^{-i a \pi}\right) \quad \text { as } \varepsilon \rightarrow 0+
$$


Hence

$$
\left(\frac{a}{2}\right)^{-1 / 2}\left(1-i e^{-i a \pi}\right) \sim-\frac{2}{h} e^{i \pi / 4} \varepsilon^{1 / 4} \frac{1+i e^{i a \pi}}{1-i e^{i a \pi}},
$$

which, on factoring out the exponentially large term $e^{i a \pi}$, gives

$$
\left(\frac{a}{2}\right)^{-1 / 2} \sim \frac{2 e^{i \pi / 4} \varepsilon^{1 / 4}}{h\left(1+i e^{-i a \pi}\right)}
$$

and hence

$$
a \sim \frac{h^{2}\left(1+i e^{-i a \pi}\right)^{2}}{2 i \sqrt{\varepsilon}},
$$

Remembering that $a=\frac{1}{2} \varepsilon^{-1 / 2} i \lambda$, where $\lambda$ is approximated by (3.4), yields

$$
\lambda \sim-h^{2}\left\{1+2 i \exp \left[\frac{\pi \lambda}{2 \sqrt{\varepsilon}}\right]\right\} \sim-h^{2}\left\{1+2 i \exp \left[\frac{-\pi h^{2}}{2 \sqrt{\varepsilon}}\right]\right\} \quad \text { as } \varepsilon \rightarrow 0+.
$$

Thus

$$
\operatorname{Im} \lambda \sim-2 h^{2} \exp \left[-\pi h^{2} / 2 \sqrt{\varepsilon}\right]
$$

\section{Computation OF THE $m(\lambda)$ FUnCtion}

As mentioned in the introduction, it is established in [4], for a general class of potentials, that the nonreal eigenvalues of the non-self-adjoint problem (1.1) correspond to the resonance poles of the associated self-adjoint problem with the usual $L^{2}(0, \infty)$ boundary condition. This result is true in general provided that the $L^{2}(0, \infty)$ solution in either the upper or lower halfplane in $\lambda$ may be shown to correspond uniquely to the outgoing wave solution. The proof is based on the work of Weyl and Titchmarsh; it uses the fact that the eigenvalues of the self-adjoint problem are given by the poles of the Green's function, which in turn correspond to the poles of the Titchmarsh-Weyl function $m(\lambda)$.

The perturbed self-adjoint problem does not admit eigenvalues, but it has a continuous spectral on the real axis (see [13, Chapter 20]). The poles of the perturbed $m(\lambda)$ function are interpreted as resonances in the sense that they correspond to regions of high spectral density; for details see [11, Chapter 12].

To follow the theory of [13], we write the constant given in (1.1) as $h=\cot \alpha$. Define fundamental solutions $\Theta(x, \lambda, \varepsilon), \Phi(x, \lambda, \varepsilon)$ of

$$
y^{\prime \prime}+\left(\lambda+\varepsilon x^{2}\right) y=0, \quad x \in(0, \infty)
$$

by

$$
\begin{array}{ll}
\Theta(0)=\cos \alpha, & \Theta^{\prime}(0)=\sin \alpha, \\
\Phi(0)=\sin \alpha, & \Phi^{\prime}(0)=-\cos \alpha .
\end{array}
$$

Titchmarsh-Weyl theory assures us that a solution which is $L^{2}(0, \infty)$ in $\operatorname{Im} \lambda>$ 0 and $\operatorname{Im} \lambda<0$ is given by

$$
\Psi(x, \lambda, \varepsilon)=\boldsymbol{\Theta}(x, \lambda, \varepsilon)+m(\lambda, \varepsilon) \Phi(x, \lambda, \varepsilon) .
$$

Knowledge of the asymptotics of the parabolic cylinder function given in $\S 2$ enables us to construct this $m(\lambda, \varepsilon)$ function as follows. We observe that, because there is a continuous spectrum on the real axis, the $m(\lambda, \varepsilon)$ functions 
will be different in the upper and lower half-planes; in what follows we take, $m(\lambda, \varepsilon)$ to be the Titchmarsh-Weyl function for $\operatorname{Im} \lambda>0$. Because we are in the Titchmarsh limit-point case, we know that the $L^{2}(0, \infty)$ solution is unique to a multiplicative constant. A fundamental set for the equation (4.1) is given by the parabolic cylinder functions $D_{-a-\frac{1}{2}}(z), D_{a-\frac{1}{2}}(-i z)$ where, as in (2.1), $a=\frac{1}{2} \varepsilon^{\frac{-1}{2}} i \lambda, z=e^{\frac{i \pi}{4}} 2^{\frac{1}{2}} \varepsilon^{\frac{1}{4}} x$. Denote their Wronskian by $w(\lambda, \varepsilon)$; this is independent of $z$ by the Abel-Liouville formula, and we shall find it convenient to evaluate it at $z=0$. Then it is easy to construct the fundamental solutions $\Theta(z, \lambda, \varepsilon), \Phi(z, \lambda, \varepsilon)$ as

$$
\begin{aligned}
w(\lambda, \varepsilon) \Theta(z, \lambda, \varepsilon)= & \left\{e^{\frac{-i \pi}{4}} \varepsilon^{\frac{-1}{4}} 2^{\frac{-1}{2}} \sin \alpha D_{a-\frac{1}{2}}(0)+i \cos \alpha D_{a-\frac{1}{2}}^{\prime}(0)\right\} D_{-a-\frac{1}{2}}(z) \\
& +\left\{\cos \alpha D_{-a-\frac{1}{2}}^{\prime}(0)-e^{\frac{-i \pi}{4}} \varepsilon^{\frac{-1}{4}} 2^{\frac{-1}{2}} \sin \alpha D_{-a-\frac{1}{2}}(0)\right\} D_{a-\frac{1}{2}}(-i z), \\
w(\lambda, \varepsilon) \Phi(z, \lambda, \varepsilon)= & \left\{i \sin \alpha D_{a-\frac{1}{2}}^{\prime}(0)-e^{\frac{-i \pi}{4}} \varepsilon^{\frac{-1}{4}} 2^{\frac{-1}{2}} \cos \alpha D_{-a-\frac{1}{2}}(0)\right\} D_{-a-\frac{1}{2}}(z) \\
& +\left\{\sin \alpha D_{-a-\frac{1}{2}}^{\prime}(0)+e^{\frac{-i \pi}{4}} \varepsilon^{\frac{-1}{4}} 2^{\frac{-1}{2}} \cos \alpha D_{-a-1 / 2}(0)\right\} D_{a-\frac{1}{2}}(-i z) .
\end{aligned}
$$

From the asymptotic behaviour of $U(a, z)$ in $(2.4)$, recalling that $D_{-a-\frac{1}{2}} \equiv$ $U(a, z)$, we observe that

$$
\begin{aligned}
D_{a-\frac{1}{2}(-i z)} & \sim e^{\frac{-(-i z)^{2}}{4}}(-i z)^{a-\frac{1}{2}} \\
& =\exp \left[\frac{i}{2} \sqrt{\varepsilon} x^{2}\right]\left(e^{\frac{-i \pi}{4}} \sqrt{2} \varepsilon^{\frac{1}{4}} x\right)^{\frac{(-\nu+i \mu)}{2 \sqrt{\varepsilon}}-\frac{1}{2}},
\end{aligned}
$$

where we have written $\lambda=\mu+i \nu, \mu, \nu \in \mathscr{R}$. We see that for $\nu=\operatorname{Im} \lambda>0$, the solution $D_{a-\frac{1}{2}}(-i z)$ is in $L^{2}(0, \infty)$ as a function of $x$ and is of outgoing wave form. Thus the eigenvalues of the non-self-adjoint problem (1.1) correspond to the resonance poles of $m(\lambda, \varepsilon)$. It remains to construct $m(\lambda, \varepsilon)$ by combining $\Theta(z, \lambda, \varepsilon)$ and $\Phi(z, \lambda, \varepsilon)$ so as to obtain a multiple of the $L^{2}$ solution $D_{a-1 / 2}(-i z)$. This leads to the choice

$$
m(\lambda, \varepsilon)=-\frac{\left\{e^{\frac{-i \pi}{4}} \varepsilon^{\frac{-1}{4}} 2^{\frac{-1}{2}} \sin \alpha D_{a-\frac{1}{2}}(0)+i \cos \alpha D_{a-\frac{1}{2}}^{\prime}(0)\right\}}{\left\{i \sin \alpha D_{a-\frac{1}{2}}^{\prime}(0)-e^{\frac{-i \pi}{4}} \varepsilon^{\frac{-1}{4}} 2^{\frac{-1}{2}} \cos \alpha D_{a-\frac{1}{2}}(0)\right\}},
$$

and we obtain

$$
\Psi(z, \lambda, \varepsilon)=\frac{-e^{\frac{-i \pi}{4}} \varepsilon^{\frac{-1}{4}} 2^{\frac{-1}{2}} D_{a-1 / 2}(-i z)}{\omega(\lambda, \varepsilon)} .
$$

The resonance poles correspond to poles of the $m(\lambda, \varepsilon)$ function that are obtained from

$$
i \sin \alpha D_{a-\frac{1}{2}}^{\prime}(0)-e^{-\frac{i \pi}{4}} \varepsilon^{\frac{-1}{4}} 2^{\frac{-1}{2}} \cos \alpha D_{a-\frac{1}{2}}(0)=0,
$$

which reduces to

$$
\frac{U(a, 0)}{U^{\prime}(a, 0)}=\frac{e^{\frac{-i \pi}{4}} 2^{\frac{1}{2}} \varepsilon^{\frac{1}{4}}\left(1+i e^{i a \pi}\right)}{h\left(1-i e^{i a \pi}\right)} .
$$

This is exactly the eigen-relation (3.2) obtained by the direct approach of $\S 3$. We have therefore illustrated the equivalence of the non-real eigenvalues of the 
non-self-adjoint problem and the resonance poles of the associated self-adjoint problem for the generalised tunnelling problem (1.1).

\section{ACKNOWLEDGMENT}

The authors wish to acknowledge a helpful suggestion from R. B. Paris for the asymptotics of the quotient of gamma functions (2.10)-(2.12).

\section{REFERENCES}

1. M. Abramowitz and I. Stegun (ed.), Handbook of Mathematical Functions, Dover, New York, 1970.

2. M. V. Berry, Uniform asymptotic smoothing of Stokes' discontinuities, Proc. Roy. Soc. London Ser. A 422, (1989) 7-21.

3. W. L. Kath and G. A. Kriegsmann, Optical tunnelling. radiation losses in bent fibre optic waveguides, IMA J. Appl. Math. 41 (1989), 85-103.

4. F. R. Lawless and A. D. Wood, Resonances and optical tunnelling, submitted.

5. C. Lozano and R. E. Meyer, Leakage and response of waves trapped by round islands, Phys. fluids, 19 No. 8, (1976), 1075-1088.

6. R. E. Meyer, Exponential asymptotics, SIAM Review 22 (1980), 213-224.

7. F. W. J. Olver, Asymptotics and special functions, Academic Press, New York, 1974.

8. _ On Stokes' phenomenon and converging factors, Asymptotic and Computational Analysis, Marcel Dekker, New York, 1990.

9. Uniform asymptotic expansions for Weber parabolic cylinder functions of large order, J. Res. Nat. Bur. Standards; B. Math. and Math. Phys. 63B, 2 (1959), pp. 131-169.

10. R. B. Paris and A. D. Wood, A model equation for optical tunnelling, IMA J. Appl. Math. 43 (1989), 273-284.

11. M. Reed and B. Simon, Methods of modern mathematical physics, Vol. 4: Analysis of operators, Academic Press, San Diego, 1978.

12. E. C. Titchmarsh, Eigenfunction Expansions. Part 1, Clarendon Press, Oxford, 1946.

13. __ Eigenfunction Expansions. Part 2, Clarendon Press, Oxford, 1958.

School of Mathematical Sciences, Dublin City University, Dublin 9, Ireland 